\title{
Transanal Total Proctocolectomy with Ileal Pouch-Anal Anastomosis for Synchronous Triple Colorectal Cancer
}

\author{
Won Jun Jeong, MD \\ Department of Surgery, Daejeon St. Mary's Hospital, College of Medicine, The Catholic University \\ of Korea, Daejeon, Korea.
}

Byung Jo Choi, MD, PhD

Department of Surgery, Daejeon St. Mary's Hospital, College of Medicine, The Catholic University of Korea, Daejeon, Korea.

\section{Sang Chul Lee, MD, PhD}

Department of Surgery, Daejeon St. Mary's Hospital, College of Medicine, The Catholic University of Korea, Daejeon, Korea.

E-mail: zambo9@catholic.ac.kr

This subject was previously presented at the International Society of University Colon and Rectal Surgeons (ISUCRS), August 29-September 1, 2018, in London, United Kingdom.

Mary Ann Liebert, Inc. DOI: 10.1089/vor.2018.0566
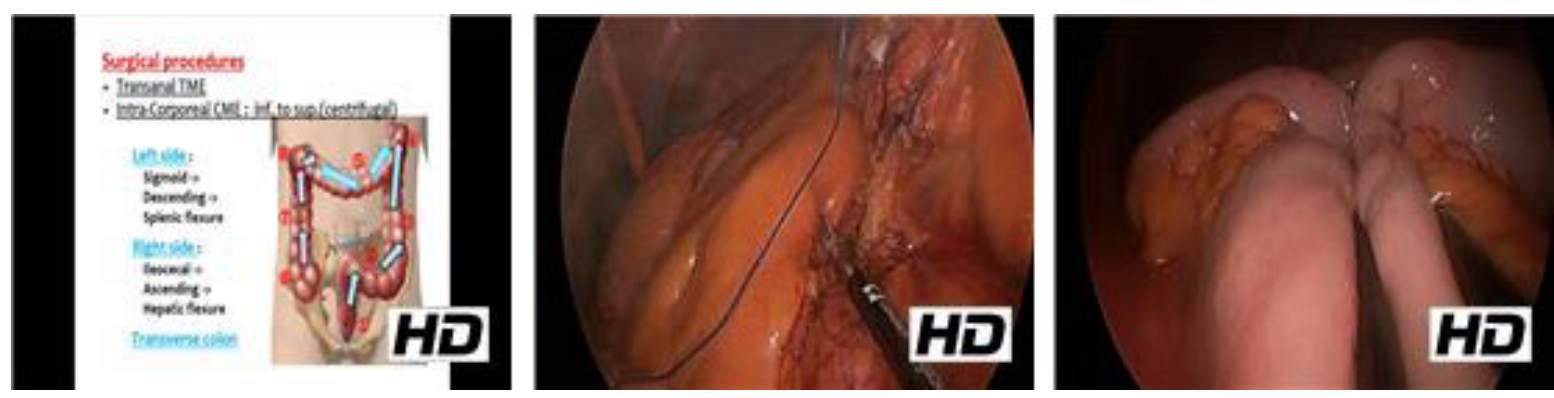

\section{Abstract}

Introduction: Transanal total mesorectal excision (TME) has been utilized as a minimally invasive surgery for colorectal cancer. ${ }^{1}$ Sylla et al. first reported the use of transanal TME and since then, various platforms have been applied for this procedure. ${ }^{2}$ The most widely used procedure is laparoscope-assisted transanal TME using a hybrid technique. de Lacy et al. introduced the Cecil procedure, which utilizes two teams (transabdominal and transanal). ${ }^{3}$ With regard to rectal cancer, a small group of authors attempted pure natural orifice transluminal endoscopic surgery (NOTES) transanal TME. ${ }^{4,5}$ The aim of this case report is to show that a transanal laparoscopic technique can be utilized for total colectomy. Except for rectal cancer, there are few reports regarding colon resection using NOTES. In this video, we perform a transanal total proctocolectomy with ileal pouch-anal anastomosis in a patient with synchronous triple colorectal cancer (ascending colon, rectosigmoid colon, and rectum).

Methods: We performed transanal total proctocolectomy with ileal pouch-anal anastomosis in a patient with synchronous triple colorectal cancer (ascending colon, rectosigmoid colon, and rectum). On preoperative MRI, there was no pelvic lateral lymph node, so we did not need to perform chemoradiation therapy. After transanal dissection of the mesorectum, rectum was flipped into the intraperitoneal space for further dissection. In our setting, we used conventional laparoscopic instruments for most procedures and long-shafted instruments helped during mobilization of the splenic and hepatic flexures. The entire specimen was extracted transanally. The ileal pouch was 
constructed intracorporeally and ileal pouch-anal anastomosis was performed using a circular stapler. We did not create a defunctioning stoma.

Results: The operating time was 328 minutes and blood loss was $<50 \mathrm{~mL}$. We harvested 61 lymph nodes, and 1 regional lymph node metastasis was found. The patient experienced temporary paralytic ileus and was discharged on postoperative day 10 and had no major complications. The patient had medications for loose stool but had no incontinence. The patient refused adjuvant chemotherapy. During the 24 months follow-up period, there were no recurrences or metastases in three colonoscopies and three CT scans. This operation was performed in February 2017 and transanal total colectomy has not been reported so far.

Conclusion: This transanal laparoscopic technique is feasible for total colectomy and may be adapted to achieve colonic resection through a natural orifice in the future.

No competing financial interests exist.

Runtime of video: 9 mins 55 secs

This subject was previously presented at the International Society of University Colon and Rectal Surgeons (ISUCRS), August 29-September 1, 2018, in London, United Kingdom.

Keywords: NOTES, synchronous colorectal cancer, transanal colectomy, total colectomy, IPAA (c) Won Jun Jeong et al. 2019; Published by Mary Ann Liebert, Inc. This Open Access article is distributed under the terms of the Creative Commons Attribution Noncommercial License (http:// creativecommons.org/licenses/by-nc/4.0/) which permits any noncommercial use, distribution, and reproduction in any medium, provided the original author(s) and the source are cited.

\section{Cite this video}

Won Jun Jeong, Byung Jo Choi, Sang Chul Lee, Transanal Total Proctocolectomy with lleal Pouch-Anal Anastomosis for Synchronous Triple Colorectal Cancer, Videoscopy. 2019, DOI: 10.1089/vor.2018.0566.

\section{References}

1. Wolthuis AM, Bislenghi G, de Buck van Overstraeten A, D'Hoore A. Transanal total mesorectal excision: Towards standardization of technique. World J Gastroenterol 2015;21:12686-12695.

2. Sylla P, Bordeianou LG, Berger D, et al. A pilot study of natural orifice transanal endoscopic total mesorectal excision with laparoscopic assistance for rectal cancer. Surg Endosc 2013;27:33963405.

3. de Lacy AM, Rattner DW, Adelsdorfer C, Tasende MM, Fernández M, Delgado S, Sylla P, Martinez-Palli G. Transanal natural orifice transluminal endoscopic surgery (NOTES) rectal resection: "down-to-up" total mesorectal excision (TME)-Short-term outcomes in the first 20 cases. Surg Endosc 2013;27:3165-3172.

4. Leroy J, Barry BD, Melani A, Mutter D, Marescaux J. No-scar transanal total mesorectal excision: The last step to pure NOTES for colorectal surgery. JAMA Surg 2013;148:226-231.

5. Zhang H, Zhang YS, Jin XW, Li MZ, Fan JS, Yang ZH. Transanal single-port laparoscopic total mesorectal excision in the treatment of rectal cancer. Tech Coloproctol 2013;17:117-123.

Original Publication Date: 2018 